\title{
IDENTIFICAÇÃO DA HIPERTENSÃO ASSOCIADA À FRAGILIDADE DO ENVELHECER E OS CUIDADOS DE ENFERMAGEM: UMA REVISÃO INTEGRATIVA
}

\author{
IDENTIFICATION OF HYPERTENSION ASSOCIATED WITH FRAILTY \\ OF AGING AND NURSING CARE: AN INTEGRATIVE REVIEW
}

\author{
Raissa Karla de Araújo Oliveira ${ }^{1}$ \\ Danielly Soares Diniz ${ }^{2}$ \\ Lindomar de Farias Belém ${ }^{3}$
}

\begin{abstract}
RESUMO: Objetivo: avaliar a relação do envelhecimento com a Hipertensão Arterial Sistêmica (HAS) e identificação de fatores contribuintes para essa morbidade, por meio de literaturas científicas disponíveis que abordem essa temática. Método: Foi realizada uma revisão integrativa da literatura, com abordagem retrospectiva, no período de 2015 a 2019. Disponíveis nas bases Pubmed - US National Library of Medicine National Institutes of Health, SciELO - Scientific Eletronic Library e Biblioteca Virtual em Saúde - BVS, por serem referências para o campo de pesquisas da Saúde. A seleção dos artigos se deu através dos critérios: atender à questão norteadora; ter disponibilidade eletrônica na forma de texto completo, ter sido publicado no período supracitado nos idiomas português ou inglês e que abordassem a população idosa. Resultados: Foram selecionados seis estudos, que atenderam aos critérios de inclusão/exclusão sugeridos. De acordo com o que foi observado após análise, o surgimentoda HAS em idosos se associa à modificações fisiológicas que acontecem no processo de envelhecimento, sendo também influenciada por fatores de risco: sedentarismo, hábitos alimentares inadequados, tabagismo e etilismo. Conclusão: O envelhecimento é um dosprincipais fatores para a HAS, além das variáveis pessoais como idade, história pregressa, e riscos modificáveis. Desse modo, embora bastante comum, a HAS ainda se configura como um dos maiores problemas de saúde pública, sendo uma temática de fundamental valor para a sociedade e profissionais de saúde, pois contribui para a ampliação do conhecimento sobre o assunto.
\end{abstract}

\footnotetext{
1 Graduanda do Curso de Enfermagem da Universidade Estadual da Paraíba - UEPB, raissakarla10@gmail.com.

2 Graduanda do Curso de Enfermagem da Universidade Estadual da Paraíba - UEPB, daniellysdiniz@hotmail.com.
}

${ }_{3}$ Doutora, Docente da Universidade Estadual da Paraíba - UEPB, lindomardefariasbelem@gmail.com. 
Palavras chave: Hipertensão. Envelhecimento. Enfermagem.

ABSTRACT: Objective: to evaluate the relationship between aging and Systemic Arterial Hypertension (SAH) and to identify factors that contribute to this morbidity, through available scientific literature that addresses this theme. Method: An integrative literature review was carried out, with a retrospective approach, from 2015 to 2019. Available at Pubmed - US National Libraryof Medicine National Institutes of Health, SciELO - Scientific Eletronic Library and Virtual Health Library - VHL, by to be references for the field of health research. The selection of articles took place through the criteria: meeting the guiding question; have electronic availability in the form of full text, have been published in the aforementioned period in Portuguese or English and that addressed the elderly population. Results: Six studies were selected, which met the suggested inclusion / exclusion criteria. According to what was observed after analysis, the appearance of SAH in the elderly is associated with physiological changes that occur in the aging process, and is also influenced by risk factors: sedentary lifestyle, inappropriate eating habits, smoking and drinking. Conclusion: Aging is one of the main factors for $\mathrm{SAH}$, in addition to personal variables such as age, past history, and modifiable risks. Thus, although quite common, SAH is still one of the biggest public health problems, being a theme of fundamental value for society and health professionals, as it contributes to the expansion of knowledge on the subject.

Keywords: Hypertension. Aging. Nursing. 


\section{INTRODUÇÃO}

Condição clínica caracterizada como multifatorial, a hipertensão arterial sistêmica (HAS) é pontuada pelo aumento dos níveis pressóricos $\geq 140$ e/ou 90 mmHg. (7 ${ }^{a}$ DIRETRIZ BRASILEIRA DE HIPERTENSÃO ARTERIAL, 2016). As doenças crônicas não transmissíveis (a qual a HAS se enquadra), são as principais fontes de carga de doença no mundo, sendo responsáveis por cerca de $13 \%$ das mortes (PELAZZA, et al., 2018).

Além disso, é estimado que a prevalência da HAS seja em torno de $30 \%$ tanto na perspectiva da população brasileira, quanto para a população mundial. Contudo, de forma particular, esse agravo atinge em grande parte a população idosa, consequência do processo natural de envelhecimento que atualmente alcança índices consideráveis (MOROZ; KLUTHCOVSKY; SCHAFRANSKI, 2016).

De acordo com o Instituto Brasileiro de Geografia e Estatística (IBGE), a população brasileira total em 2018 é de 208.494 .900 milhões de pessoas, onde $12,6 \%$ se referem a pessoas com 60 anos ou mais. Essa atual configuração demográfica se refere à diminuição da taxa de fecundidade, assim como o aumento da expectativa de vida. A transição na progressão do crescimento populacional em caráter mundial é um fator desafiante para a assistência da população idosa, visto que a expectativa é que em 2025 exista 1,2 bilhões de pessoas acima de 60 anos (FERREIRA, et al., 2017; ARAUJO, et al., 2018; CAVALCANTI, et al., 2019).

A associação direta e linear entre o envelhecimento e a prevalência de HAS está relacionada à: i) aumento da expectativa de vida da população brasileira, que se encontra atualmente em 74,9 anos; ii) aumento da população de idosos $\geq 60$ anos na última década (2000 a 2010). Dessa forma, em meio aos conhecimentos do problema e com a meta-análise de estudos realizados no Brasil incluindo 13.978 indivíduos, ficou evidenciada a predominância de $68 \%$ de HAS em idosos $\left(7^{\text {a }}\right.$ DIRETRIZ BRASILEIRA DE HIPERTENSÃO ARTERIAL, 2016). 
Considerar a fisiologia do envelhecimento do organismo é fator preponderante para assimilar a regressão das funcionalidades cardiovasculares, assim como suas consequências clínicas (PELAZZA, et al., 2018). O envelhecimento vascular é o fator principal relacionado à elevação da pressão arterial (PA) nos idosos. Além disso, a microarquitetura dos vasos e consequente enrijecimento arterial possibilita que grandes vasos, como a aorta, perca sua distensibilidade. Clinicamente, a rigidez da parede das artérias se expressa como Hipertensão Sistólica Isolada (HSI), condição com grande prevalência na população geriátrica (7 $7^{a}$ DIRETRIZ BRASILEIRA DE HIPERTENSÃO ARTERIAL, 2016).

As alterações decorrentes do envelhecimento acabam tornando o idoso vulnerável ao processo de morbimortalidade. Dessa forma, as doenças crônicas não transmissíveis (DCNT) são bastante recorrentes nessa população. Por existir relação direta e linear da PA com a idade, observa-se que esse fator provoca diversas mudanças no indivíduo, na família, na sociedade e no sistema de saúde. A avaliação e diagnóstico da HAS, quando feita de forma incorreta, acaba resultando em uso abusivo de medicações, fazendo com que o paciente desenvolva efeitos colaterais que prejudicam a qualidade de vida (PELAZZA, et al., 2018).

De acordo com as condições já citadas, os idosos apresentam maiores necessidades acerca da assistência em saúde, sobretudo, da equipe de enfermagem. Dessa forma, responder essa situação de forma adequada, respeitando o princípio da equidade, é uma preocupação significativa de toda equipe de saúde (BAO; TANG; CHEN, 2017).

Nesse aspecto, em 2006, foi instituído a Política Nacional de Saúde à Pessoa Idosa (PNPI) pelo Ministério da Saúde, garantindo a promoção, prevenção e recuperação da saúde aessa população. Essa realidade conferiu ao profissional de saúde, sobretudo ao enfermeiro, uma parcela bastante importante acerca da assistência prestada (ARAUJO, et al., 2018). A redução da morbimortalidade é resultado não só do controle da PA, mas também da adesão dos profissionais de saúde às ações institucionais e ao atendimento multiprofissional (MOROZ; KLUTHCOVSKY; SCHAFRANSKI, 2016).

O objetivo do presente estudo foi avaliar a relação do envelhecimento com a HAS, a identificação de fatores contribuintes relacionados à ocorrências desta 
morbidade, através da literatura cientifica, correlacionando à descrição dos resultados encontrados no enfoque da atuação da enfermagem. Todavia, é ensejado ampliar as discussões sobre o assunto discutido sendo este pouco elucidado pela literatura.

\section{MÉTODO}

Para alcançar o objetivo, foi realizada uma revisão integrativa da literatura, que atendesse a compreensão da natureza a ser pesquisada, gerando um levantamento de dados precisos e atuais de problemas e/ ou teorias fundamentais para a área científica; através da elaboração da pergunta norteadora; seleção/ busca (critérios de inclusão e exclusão); coleta de dados e análise crítica; discussão dos resultados e apresentação da revisão integrativa.

Para tal, a questão norteadora desta pesquisa é: Qual a relação do envelhecimento com a hipertensão? A seleção dos artigos se deu por meio de busca das publicações da literatura científica, no período de Janeiro de 2015 a dezembro de 2019 nos idiomas inglês e português, na base de dados da Biblioteca Virtual em Saúde (BVS), Scielo (Scientific Electronic Library Online) e PubMed (US National Library of Medicine National Institutes of Health). Na BVS foi possível realizar uma busca simultânea das publicações relevantes nas principais bases de dados científicos no campo nacional e internacional.

Os critérios de inclusão estabelecidos para a seleção dos artigos foram: Responder a questão norteadora; ter disponibilidade eletrônica na forma de texto completo, ter sido publicado no período supracitado nos idiomas português ou inglês e estudos que abordavam a população idosa nos quesitos: a) cuidados de enfermagem a idosos com hipertensão; b) vulnerabilidade e fatores que possam afetar no processo saúde-doença; c) cuidados da equipe de saúde no controle da morbidade.

Foram excluídos: Estudos repetidos em uma ou mais bases de dados, estudos que não abordassem a população idosa ou perspectivas que não se 
adequassem à questão norteadora. Esses critérios foram utilizados tanto para a leitura de resumos, como para a leitura do texto completo. Para a busca dos artigos foram utilizadas três palavras chaves indexadas nos Descritores em Ciências da Saúde (DeCS): Hipertensão (hypertension), envelhecimento (Aging); e enfermagem (Nursing); foram realizadas todas as possibilidades de cruzamento entre os descritores selecionados, utilizando o operador boleano "and".

O período de busca dos artigos deu-se no fim de dezembro de 2019 e concluiu-se no início de janeiro de 2020. A análise e a síntese dos dados extraídos dos artigos foram realizadas de forma descritiva, no Microsoft Word 2013, de forma a reunir os resultados necessários à questão a ser pesquisada.

\section{RESULTADOS E DISCUSSÃO}

A pesquisa nas bases de dados mediante o cruzamento entre todos os descritores resultou em 324 artigos, os quais passaram por uma pré-seleção através da leitura dos títulos e dos resumos, quando necessário. Entretanto, ao término dessa fase foram pré-selecionados 94 artigos conforme critérios de inclusão e exclusão mencionados. Desses, após leitura somente do título foram excluídos 59 , restando apenas 35 artigos. Em seguida, decorrido a leitura dos resumos, restaram apenas 15 e para finalização através da leitura na íntegra, de modo a identificar a adequação dos mesmos ao assunto a ser pesquisado, foram excluídos 9, chegando-se a uma amostra final de 6 artigos em que estão apresentados no Quadro 1, sendo um na base Pubmed, dois na Scielo e três na BVS, conforme demonstrado em Fluxograma 1.

Para a Análise dos dados, foi utilizado um instrumento de coleta de dados para revisões integrativas, utilizando as seguintes variáveis: ordem, data, título, metodologia e objetivos. 
Fluxograma 1 - Fluxograma da pesquisa de artigos nas bases de dados.

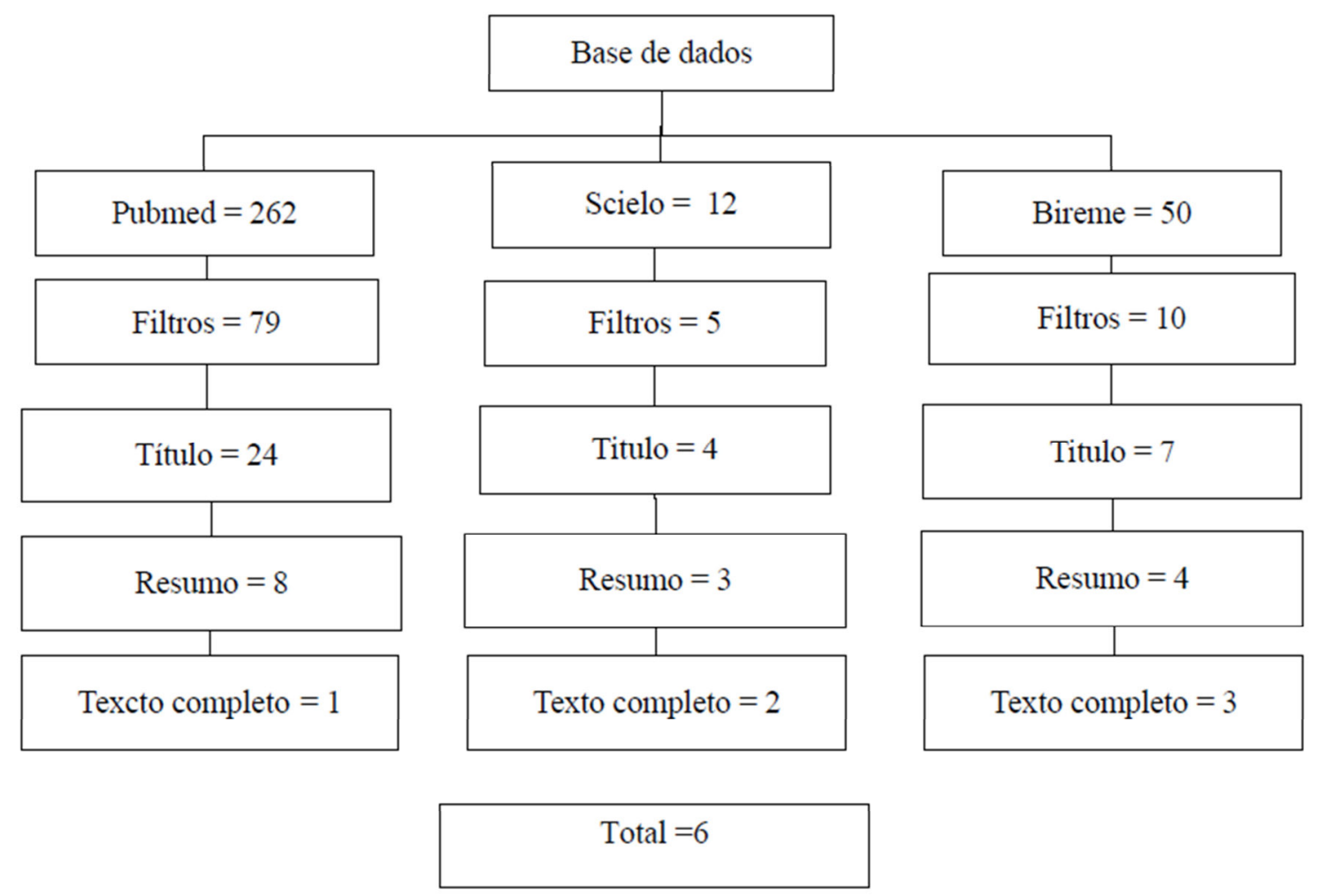

Tabela 1 - Descrição dos artigos selecionados, quanto à ordem, título, base de dados, periodico, país, dioma e temática, de 2015 - 2019.

\begin{tabular}{|c|c|c|c|c|c|}
\hline $\mathbf{N}^{\mathbf{O}}$ & Título & $\begin{array}{c}\text { Base de } \\
\text { dados }\end{array}$ & Períodico & País/ Idioma & Temática \\
\hline 01 & $\mid \begin{array}{ll}\text { Individual } \\
\text { nursing } & \text { care for } \\
\text { the } & \text { elderly } \\
\text { among } & \text { China's } \\
\text { aging } & \\
\text { population }\end{array}$ & Pubmed & $\begin{array}{l}\text { BioScience } \\
\text { Trends. 2017; } \\
\text { 11(6):694-696. }\end{array}$ & China/Inglês & $\begin{array}{lr}\text { Mostrar } & \text { a } \\
\text { necessidade } & \text { de } \\
\text { cuidados } & \\
\text { individualizados } & \\
\text { para cada tipo } & \text { de } \\
\text { idade } & \text { ou } \\
\text { necessidade. } & \\
\end{array}$ \\
\hline 02 & $\begin{array}{l}\text { Hábitos de vida } \\
\text { de homens } \\
\text { idosos } \\
\text { hipertensos }\end{array}$ & Scielo & $\begin{array}{l}\text { Rev Gaúcha } \\
\text { Enferm. } \\
\text { 2019;40:e20180 } \\
115\end{array}$ & $\begin{array}{l}\text { Brasil/ } \\
\text { Português }\end{array}$ & $\begin{array}{l}\text { Analisar quais são } \\
\text { os hábitos de vida } \\
\text { que a população } \\
\text { idosa } \\
\text { aderindo. }\end{array}$ \\
\hline
\end{tabular}




\begin{tabular}{|c|c|c|c|c|c|}
\hline 03 & $\begin{array}{lr}\text { Controle } & \text { da } \\
\text { pressão arterial } \\
\text { em } & \text { idosas } \\
\text { hipertensas } & \text { em } \\
\text { uma } & \text { Unidade } \\
\text { de Saúde } & \text { da } \\
\text { Família } & \text { e } \\
\text { fatores } & \\
\text { associados }\end{array}$ & Scielo & $\begin{array}{l}\text { Cad. Saúde } \\
\text { Colet., 2016, Rio } \\
\text { de Janeiro, } 24 \\
\text { (1): } 111-117\end{array}$ & $\begin{array}{l}\text { Brasil/ } \\
\text { Português }\end{array}$ & $\begin{array}{lr}\text { Analisar o } & \text { controle } \\
\text { da HAS em } & \text { um } \\
\text { grupo de idosas } & \text { de } \\
\text { uma USF } & \text { e } \\
\text { enfatizar } & \text { a } \\
\text { impotância } & \text { do } \\
\text { controle } & \text { para } \\
\text { redução } & \text { de } \\
\text { morbimortalidades. }\end{array}$ \\
\hline 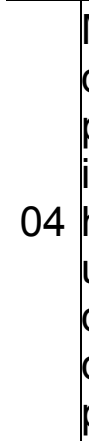 & 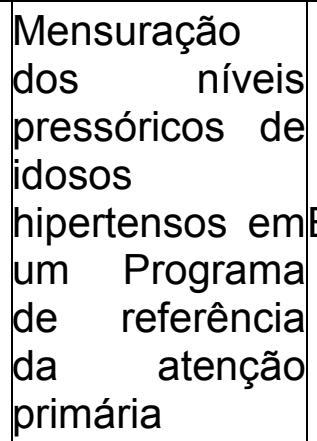 & BVS & $\begin{array}{l}\text { Rev enferm } \\
\text { UFPE on line., } \\
\text { Recife, } \\
12(2): 364-70, \\
\text { fev., 2018 }\end{array}$ & Brasil/ & $\begin{array}{lr}\text { Buscar através de } \\
\text { consultas } & \text { analisar } \\
\text { os r níveis } \\
\text { pressóricos r em } \\
\text { idosos hipertensos. }\end{array}$ \\
\hline 05 & \begin{tabular}{|lr}
\multicolumn{3}{|l|}{ Caracterização } \\
da saúde & de \\
idosos & \\
Cadastrados & \\
em & uma \\
unidade & de \\
saúde & Da \\
família & \\
\end{tabular} & BVS & $\mid \begin{array}{ll}\operatorname{Rev} & \text { baiana } \\
\text { enferm } & (2018) ; \\
32: \text { e28041 }\end{array}$ & $\begin{array}{l}\text { Brasil/ } \\
\text { Português }\end{array}$ & $\begin{array}{l}\text { Estudar como é a } \\
\text { saúde de idosos } \\
\text { emum USF, onde a } \\
\text { maioria apresnta } \\
\text { HAS. }\end{array}$ \\
\hline 06 & $\begin{array}{l}\text { Fatores } \\
\text { risco de } \\
\text { doenças } \\
\text { cardiovasculare } \\
\text { s emidosos }\end{array}$ & BVS & $\begin{array}{l}\text { Rev enferm } \\
\text { UFPE on line., } \\
\text { Recife, } \\
11(12): 4895- \\
905, \text { dec., } 2017\end{array}$ & Brasil/ & $\begin{array}{l}\text { Observar e } \\
\text { identificaros fatores } \\
\text { para surgimento de } \\
\text { doenças } \\
\text { cardiovasculares } \\
\text { nos idosos. }\end{array}$ \\
\hline
\end{tabular}

Por conseguinte, na base PubMed foram encontrados (1) 100\% em língua inglesa, já na SciElo foram encontrados (2) 100 \% na língua portuguesa e na BVS (3) $100 \%$ na mesma língua supracitada. Com relação ao ano, desses seis artigos encontrados, um era de 2016: $16,6 \%$, dois de 2017 : $33,3 \%$, dois de $2018: 33,3 \%$ e um de 2019: 16,6\%.

Já com foco nas revistas publicadas percebeu-se que a mais divulgada é a Revista de enfermagem da Universidade Federal de Pernambuco, com 33,3\% (2), tendo mais três brasileiras: Revista Gaúcha de enfermagem, Revista Baiana de 
enfermagem e o Caderno de saúde coletiva do Rio de Janeiro, as quais resultaram em 16,6\% cada uma, além de uma internacional, em 16,6\% (1).

Em relação à metodologia, a abordagem quantitativa resultou no total de seis artigos, totalizando em 100\%. Quanto ao desenho metodológico foram variados, sendo eles estudos exploratórios pesquisa de campo tendo característica descritiva. De acordo com o que foi observado nos estudos, a população participante das pesquisas em grande maioria era feminina em 50\%, embora um estudo se dedicasse exclusivamente a esse público 16,6\%, e um estudo se dedicasse ao público masculino 16,6\%. Nesse aspecto, a população avaliada tinha de 60 anos ou mais em (5) 83,3 \% dos estudos.

Com a leitura dos resultados das pesquisas selecionadas nesta revisão, observa-se que a relação da HAS com o envelhecimento é um fator bastante relevante. O estudo 1, realizado mediante dados da "China Health and Family Planning Statistical Yearbook 2016", descreve a prevalência de HAS em idosos chineses cerca de seis vezes mais que em pessoas entre as faixas etárias de 18 a 44 anos; esse dado fica ainda mais evidenciado no fato de que essa população está mais propensa a adquirir doenças crônicas, chegando geralmente, de 2 a 3 condições diferentes.

O estudo 2, realizado com homens idosos hipertensos da cidade de Currais Novos - Rio Grande do Norte, evidencia que há relação direta entre a PA e a idade, visto que a partir dos 60 anos os níveis pressóricos tendem a aumentar; atrelado a isso, a presença de doenças crônicas, além das pessoas acima de 65 anos ocuparem a faixa etária de menor diminuição do tabagismo no Brasil, se configuram como fatores contribuintes para a prevalência de HA em idosos (CAVALCANTI, et al., 2019).

No estudo 3, realizado com idosas hipertensas em uma Unidade de Saúde da Família (USF), localizada na região sul do Brasil, o mesmo é observado. Assim sendo, a condição destacada nessa pesquisa com associação ao aumento dos níveis pressóricos em idades mais avançadas, é a maior dificuldade de normalização da PA. Esse fato é evidenciado a partir do estudo Controlar Brasil, que alega que para cada ano de vida reduz em $2 \%$ a probabilidade de controle da PA. Além disso, outros fatores são atribuídos como agravantes para o caso, comoa falta 
de comparecimento às consultas médicas e ao uso de medicamentos de forma irregular (MOROZ; KLUTHCOVSKY; SCHAFRANSKI, 2016).

O estudo 4 foi realizado com idosos hipertensos cadastrados em um programa de educação e controle de HAS em um município do sudoeste goiano; os resultados evidenciaram a regressão das propriedades cardiovasculares com o envelhecimento (modificações das paredes arteriais, enrijecimento devido a diminuição da elastina e colágeno) como fatores precursores da elevação da Pressão Arterial Sistólica (PAS). Dessa forma, a aferição da Pressão de Pulso (PP) apresentou relevância da primeira para a segunda aferição, representando o aumento gradativo do Risco Cardiovascular (RCV) e sua associação ao caso (PELAZZA, et al., 2018).

O estudo 5 foi realizado com idosos cadastrados em uma Unidade Básica de Saúde (UBS) do sítio Wanderley, no município de Recife, Pernambuco. Os resultados evidenciam as percas naturais (fisiológicas) do envelhecimento, como consequência da vulnerabilidade dessa faixa etária à doenças, sendo a mais relatada a HAS (ARAUJO, et al., 2018).

Por fim, o estudo 6 foi realizado com 246 idosos atendidos em 14 centros de referências da assistência social de 14 municípios de Maciço de Baturité. Os resultadosmostraram as diversas condições crônicas e as escolhas de estilo de vida (tabagismo, etilismo, comportamento sexual de risco, hábitos alimentares inadequados e sedentarismo) comuns ao envelhecimento, predispondo a HAS da população estudada e afetando a qualidade de vida do idoso (FERREIRA, et al., 2017).

De modo geral, dentre os riscos modificáveis, o mais apontado foi o sedentarismo (4) 66,6\%, tabagismo (3) $50 \%$, seguido do etilismo (2) $33,3 \%$. Dessa forma, faz-se importante que a equipe de saúde observe e atue de forma efetiva nesse fato, visto que estudos apontam que indivíduos ativos apresentam risco $30 \%$ menor de desenvolver HAS que os sedentários, e o aumento da atividade física reduz a PA (7 $7^{\text {a }}$ DIRETRIZ BRASILEIRA DE HIPERTENSÃO ARTERIAL, 2016).

$O$ tabagismo sendo um fator recorrente nos estudos apresenta-se como um grande problema de saúde pública, visto que há relação com a hipertensão, além de ser a principal causa de morte evitável. Não obstante, essa prática juntamente com o 
etilismo são mais comuns em homens, deixando assim essa população mais susceptível ao caso (FERREIRA, et al., 2017).

E esse é um fator preocupante, visto que a busca dos homens pelas assistências de saúde é bem menor, sendo a incompatibilidade de horário do trabalho versus funcionamento dos serviços como principal motivo apontado (CAVALCANTI, et al., 2019).

Assim, segundo Cavalcanti, et al. (2019) foi observado após pesquisa que a privação ao tabagismo influenciou de forma positiva para a não ocorrência de picos hipertensivos, reforçando a importância da redução desse hábito para o controle dos níveis pressóricos.

Ademais, o etilismo quando praticado em excesso, corrobora com efeitos deletérios no organismo do idoso, visto que o envelhecimento provoca alterações no metabolismo do álcool tornando-o mais propenso à intoxicação (FERREIRA, et al., 2017).

Outro problema relevante, presente em um estudo $16,6 \%$, é a falta de controle da PA, acusando a adesão ao tratamento da HAS um fator deficiente. Nesse aspecto, observa-se a necessidade de estudos para a garantia da adesão do idoso ao tratamento prescrito assim como o controle devido. Como problema desencadeador desse fato, dois artigos: 33,3\% apontam o baixo índice de educação escolar como um fator dificultante da compreensão das orientações de saúde passada pela equipe.

Dessa forma, profissionais de saúde tem o papel importante em manter um olhar holístico, introduzindo o idoso na escolha de recursos terapêuticos e orientando de forma simples e adequada a importância de manter um estilo de vida saudável, com a prática de atividades físicas, alimentação adequada, adesão ao tratamento medicamentoso, diminuição do tabagismo e etilismo (PELAZZA, et al., 2018).

Ademais, um fator que chama a atenção, embora presente em um estudo $16,6 \%$ é a presença de um considerável percentual de idosos com níveis pressóricos alterados quedesconheciam essa situação.

Nesse aspecto, em $66,6 \%$ dos estudos a enfermagem é comtemplada com um papel importante em ações pautadas para a promoção, contemplando 
educações em saúde, assim como o controle, diagnóstico precoce da HAS e acompanhamento devido aos idosos. Dessa forma, para uma assistência efetiva, faz-se importante a realização do diagnóstico da população que recorre ao atendimento profissional, para que as necessidades sejam contempladas. Além disso, a implantação de políticas públicas e serviços que tenham como foco as doenças crônicas são ótimos fatores para promover e garantir a autonomia, o bemestar e a qualidade de vida do idoso (FERREIRA, et al., 2017; ARAUJO, et al., 2018).

\section{CONSIDERAÇÕES FINAIS}

O número da população idosa ocupa níveis cada vez mais elevados. Diante dos achados é possível relatar que a HAS está diretamente associada a alterações fisiológicas que ocorrem no processo do envelhecimento, onde as artérias passam por perdas naturais, deixando de funcionar adequadamente, causando alteração do fluxo sanguíneo. Bem como também por variáveis pessoais como: idade, história pregressa, e riscos modificáveis (prática do tabagismo, etilismo, sedentarismo, hábitos alimentares inadequados). Desse modo, embora bastante comum, a HAS ainda se configura como um dos maiores problemas de saúdepública.

Nessa circunstância, faz-se necessário que um maior conhecimento esteja relacionado a esse aspecto, não só incluindo a sociedade de modo geral, mas também os profissionais da saúde. Esse fato é evidenciado na pequena quantidade de estudos atuais selacionados.

Outro fator preponderante é a presença de pequena quantidade de artigos que enquadrem a enfermagem, nessa perspectiva. Diante disso, identificou-se que há necessidade do fortalecimento das políticas públicas e das ações como o hiperdia nas Unidades Básicas de Saúde e em Unidades de Estratégia de Saúde da Família, além de ações para a promoção de saúde e prevenção de riscos voltados a esse público alvo. E os profissionais tem papelfundamental nisso, 
visto que os mesmos conhecem a realidade do público, além de ter uma relação direta para estimular o cuidado integral.

Além disso, o trabalho torna-se importante para promover o maior nível de conhecimento cientifico para formação destas, assim como o maior aporte teórico para profissionais agirem na prevenção e cuidados gerais de saúde.

\section{REFERÊNCIAS BIBLIOGRÁFICAS}

ARAÚJO, G. K. N. de et al. CARACTERIZAÇÃO DA SAÚDE DE IDOSOSCADASTRADOS EM UMA UNIDADE DE SAÚDE DA FAMÍLIA. Revista Baiana de Enfermagem, [s.l.], v. 32, p.111, 23 jan. 2019.

BAO, J.; TANG, Q.; CHEN, Y.. Individual nursing care for the elderly among China's aging population. Bioscience Trends, [s.I.], v. 11, n. 6, p.694-696, 2017.

CAVALCANTI, M. V. de A. et al. Hábitos de vida de homens idosos hipertensos. Revista Gaúcha de Enfermagem, [s.I.], v. 40, 18 fev. 2019.

FERREIRA, J. D. F. et al. Fatores de risco para doenças cardiovasculares em idosos. Revista de Enfermagem UFPE On Line, [s.I.], v. 11, n. 12, p.4895-4905, 4 dez. 2017.

MALACHIAS M. V. B et al. $7^{\text {a }}$ Diretriz Brasileira de Hipertensão Arterial. Arq Bras Cardiol 2016; 107(3Supl.3):1-83.

MOROZ, M. B.; KLUTHCOVSKY, A. C. G. C.; SCHAFRANSKI, M. D. Controle da pressão arterial em idosas hipertensas em uma Unidade de Saúde da Família e fatores associados. Cadernos Saúde Coletiva, [s.I.], v. 24, n. 1, p.111-117, mar. 2016.

PELAZZA, B. B. et al. Mensuração dos níveis pressóricos de idosos hipertensos em um programa de referência da atenção primária. Revista de Enfermagem UFPE On Line, [s.I.], v. 12, n. 2, p.364-370, 4 fev. 2018. 